\title{
Antenna Design Using Genetic Algorithms
}

\author{
Derek S. Linden \\ Linden Innovation Research \\ PO Box 1601 \\ Ashburn VA 20146-1601 \\ dlinden@lindenir.com
}

\begin{abstract}
Antennas are an important component in any wireless system, as they transform a signal that flows through wires into a signal that propagates through space and back again. How well it does this job is a determining factor in how well a wireless system will operate. Two real-world problems are described in this paper that are solved by antennas optimized by genetic algorithm. The antennas show the ability of the genetic algorithm to allow the designer to optimize an antenna for several different criteria at once, and to create new antennas with very little information from the designer other than general constraints and the desired performance characteristics.
\end{abstract}

\section{INTRODUCTION}

Communication, radar and remote sensing systems employ thousands of different types of antennas, and there is an increasing need for them to be highperformance and customized. Traditional methods of designing and optimizing antennas by hand using simulation or analysis are time- and labor-intensive, and limit complexity. Local search techniques are helpful, but because the search spaces of even simple antennas are highly multimodal, the initial guess must be close to the final design, and therefore these methods have limited usefulness.

Evolutionary computation methods like the genetic algorithm (GA) are able not only to optimize performance of existing antenna designs, but also to create new kinds of antennas with highly counterintuitive designs. Using a $\mathrm{GA}$, it is possible to prescribe the desired performance of an antenna and allow the computer to find the parameters for the design.
GAs are being applied to many different antenna designs by many different researchers [1]. GAs and other evolutionary computation techniques are very useful in this field for several reasons, including:

- Antenna principles, which are a subset of electromagnetics and founded on Maxwell's equations, are extremely difficult to understand and grasp intuitively.

- There are many fast antenna simulators, requiring only seconds to produce accurate results.

- Search spaces are highly multimodal and resistant to other forms of numerical and hands-on optimization, yet finding good designs is important to industry.

- Since the GA is naturally robust to local optima, and does not even require an initial guess, the amount of design information the engineer must supply to get a good result is minimal.

The GA has the ability to find new solutions when no known conventional antenna designed with conventional techniques is able to approach the requirements of a particular problem, or when such an antenna is expensive and/or difficult to manufacture.

Most antenna optimizations begin with a conventional design and the GA finds the optimal parameters based on desired conventional characteristics. For instance, an inherently high-directivity design like the Yagi-Uda antenna may be optimized for maximum gain (these terms are defined in the next section). This approach is certainly useful, since even conventional problems are difficult to optimize with most other methods, and the resulting optimized designs will often be better than any found previously.

However, of greater interest is to apply conventional designs to unconventional applications, where the GA has enough degrees of freedom to significantly change the mode of operation of the antenna to suit the new application, and to create new antennas when the amount of engineering constraint is minimal. 
As the world goes increasingly wireless, there is a growing number of antenna problems without good solutions. The tracking of hospital patients, biomedical research, wideband data communications, remote sensing, integration of antennas within electronic devices, and many others, are all demanding antennas that meet their needs. Meeting them rapidly and effectively will require new approaches to antenna design, because the traditional method is too limited to keep pace with the rising demand. What the GA provides is a means to explore areas of antenna design previously unsearchable and solve antenna design problems unhindered by the limits of human intuition and experience.

\section{ANTENNA BASICS}

This section provides a brief tutorial on antenna design concepts that will help the reader to understand the designs described in later sections.

There are many antenna classes, such as reflector antennas (e.g., dish antennas), phased array antennas (consisting of multiple regularly spaced elements), wire antennas, horn antennas, and microstrip and patch antennas. Each of these classes use different structures and exploit different properties of electromagnetic waves. Wire antennas will be the focus of this paper. An antenna is a wire antenna if it is constructed from conductors that are much longer than their width.

A ground plane-at its simplest a large, flat piece of metal underneath the antenna-is often used in conjunction with a wire antenna. It acts as a mirror for the antenna above it, and therefore changes the antenna gain pattern. A ground plane can decrease the required height and/or simplify the construction of the wire antenna. The hood or roof of a car acts as a ground plane, and antennas that will be affixed to such places need to be designed for use with one.

Directivity and gain are two related qualities in antenna design. Directivity is the ratio of power density being transmitted by an antenna in a particular direction to the average power density being transmitted in all directions. The gain is the directivity multiplied by the ratio of power radiated to power input. Gain takes into account all losses such as loss due to resistance in the antenna, which converts some of the input power into heat, and loss due to mismatch between the transmitter/receiver and the antenna. When the losses are considered to be zero, as in this chapter, the directivity and gain are equal.

Gain is usually expressed in decibels $(\mathrm{dB})$, which relates to a ratio of power or power densities by the following expression: $\mathrm{dB}=10 \log _{10}\left(\mathrm{P}_{1} / \mathrm{P}_{2}\right)$. In the case of gain, $\mathrm{P}_{2}$ is the power density of an isotropic radiator that transmits power equally in all directions. The abbreviation $\mathrm{dBi}$ refers to gain compared with an isotropic radiator. However, the " $\mathrm{i}$ " is sometimes left off, and is understood from context.
A gain pattern or antenna pattern plots gain magnitude versus angle, showing the proportion of power an antenna transmits in a particular direction. For 2-D antennas, or antennas symmetric in the third dimension, this angle is simply the elevation angle $\theta$. In 3-D, there are two angles that specify a direction: $\theta$ and the azimuth $\phi$. Figure 1 shows these angles on a set of axes. An antenna is considered to be directive if its gain pattern is heavily weighted in one direction. The greater the desired directivity, the larger the antenna must be relative to a wavelength, which is commonly labeled $\lambda$. The wavelength is the speed of light divided by the frequency, so a $300 \mathrm{MHz}$ signal has a wavelength of about $1 \mathrm{~m}$.

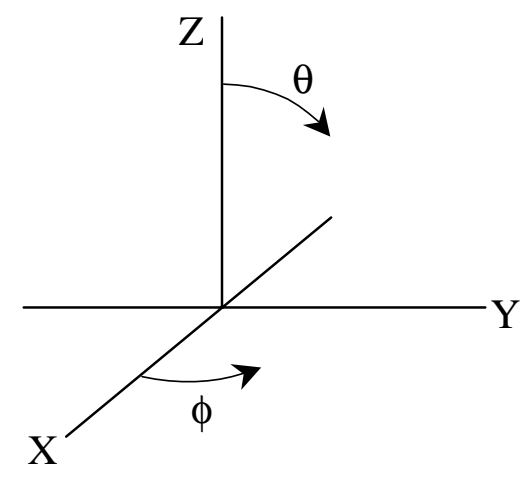

Figure 1: $\theta$ and $\phi$ on a 3-D Axis System

An antenna's beamwidth refers to the useful angle span of the so-called main lobe or beam. This lobe usually has the highest gain in the pattern, and is what is of interest to optimize. In a uniform gain pattern, there is only one lobe, but in a directive pattern where the beamwidth is desired to cover only a certain angle span, there can exist other lobes. These other lobes are called sidelobes, and usually the designer seeks to minimize them.

Voltage Standing Wave Ratio, or VSWR, is a way to quantify the match between an antenna and a device connected to it. A standing wave is created when there is a mismatch in this connection, which prevents power from flowing to and from the antenna. If the standing wave is large, implying a high VSWR, there is a significant mismatch. If it is low, the match is good. A VSWR of 3.0 or less is considered adequate for many low-power applications, while a VSWR less than 1.5 or 2.0 is desired if power considerations are important. A VSWR of 1.0 is a perfect match, and it can never be less than 1. VSWR is easy to measure, and since it is a common parameter specified by antenna designers, it is often an important quantity to optimize.

Bandwidth is the useful range of frequencies for an antenna, and is usually desired to be as large as possible. It is given in percent, which is the ratio of the useful frequency span over the nominal operating frequency. For an antenna operating at $2 \mathrm{GHz}$, a bandwidth of $3 \%$ would 
mean it would operate over a $60 \mathrm{MHz}$ range, from $1.97 \mathrm{GHz}$ to $2.03 \mathrm{GHz}$.

Polarization refers to the orientation of electromagnetic waves. Electromagnetic waves are composed of two components: an E-field (electric field) component, which is a sinusoidal wave that exists in one plane, and an $\mathrm{H}-$ field (magnetic field) component that exists at rightangles to the E-field. Thus, the wave is asymmetric, and has a definite orientation. Since the $\mathrm{H}$ field is constrained by the E-field in a propagating wave, we will discuss just the E-field. In a wave of constant overall magnitude, the E-field magnitude can actually be a time-varying quantity. If one looks at a wave propagating past a fixed point in space, the E-field can oscillate back and forth in a single orientation, giving linear polarization, or it can actually be rotating in a circle as its $\mathrm{x}$ and y components oscillate back and forth out of phase, giving circular polarization. It can also take an orientation in between, giving elliptical polarization. Antennas are polarization-sensitive: an antenna that is optimal for picking up linear polarized signals will miss half of the energy of a wave that is circularly polarized and all of a wave that is crosspolarized (linearly polarized at a right angle to the antenna). An antenna that is left-hand circularly polarized (the E-field moves in a circle to the left) will miss a righthand circularly polarized wave completely. In addition, for ground-to-satellite communications, circular polarization is very helpful because it minimizes the effect of polarization distortion that occurs as a signal travels through the ionosphere.

The next section discusses a conventional design optimized for an unconventional application.

\section{A CONVENTIONAL DESIGN AND AN UNCONVENTIONAL APPLICATION: THE YAGI-UDA ANTENNA}

As shown in the figure below, the Yagi-Uda antenna is a series of parallel wires, first proposed by Prof. Yagi and his student S. Uda in the late 1920s. One element is driven, one element is behind the driven element and is called the reflector, and all other elements are called directors. The highest gain can be achieved along the axis and on the side with the directors. The reflector acts like a small ground plane, allowing power that would otherwise be sent backward to be reflected forward.

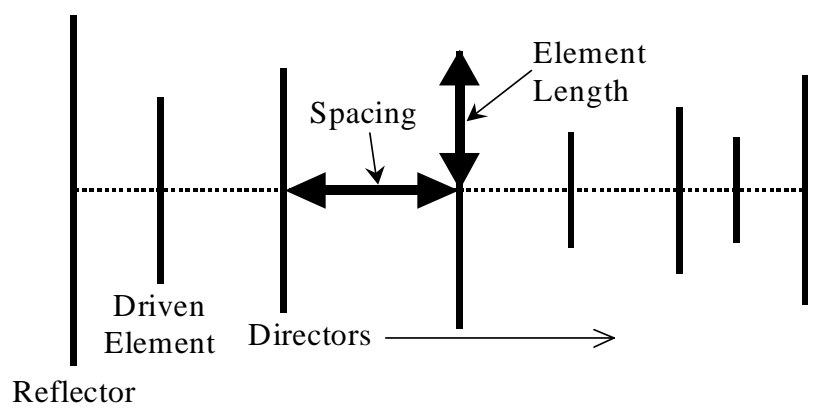

Figure 2: The Yagi-Uda Antenna

The conventional Yagi design includes geometry variables of length for each element, spacing between elements, and the diameter of the wire. Thus, with $\mathrm{N}$ elements, there are $\mathrm{N}$ length variables, N-1 spacing variables, and one wire diameter variable, giving $2 \mathrm{~N}$ variables total. The center of the driven element is where the feed is connected.

An unconventional application of this antenna is described in [3], and involves designing a special antenna for the Arecibo 305-meter spherical reflector in Puerto Rico [4]. The antenna was to be used to search for primeval hydrogen having a redshift of approximately 5 . Neutral hydrogen line emission is at a frequency of 1420 $\mathrm{MHz}$; thus the frequency region of interest was about 235 MHz. Preliminary studies indicated that the band from 219 to $251 \mathrm{MHz}$ was of the greatest interest, particularly from 223 to $243 \mathrm{MHz}$. The most important design goal was for the feed to have sidelobes at least $25 \mathrm{~dB}$ down from the main beam gain in the region from $70^{\circ}<\phi<$ $290^{\circ}$, due to the interference which came from surrounding radio and TV towers. Of lesser importance was that the E-plane (the plane parallel to the plane of the antenna) and H-plane (perpendicular to the E-plane) beamwidths be about $50^{\circ}$. The VSWR was desired to be under 3.0 and the gain was to be as high as possible, limited by the wide beamwidth. The feed would be mounted over a 1.17 meter square ground plane - that is, a ground plane only $0.92 \lambda$ in size. The antenna also needed to have a single polarization so that two of them operated at cross-polarity (i.e., each would have a polarization exactly opposite the other), and thus could be used to discriminate between the randomly polarized hydrogen signal and the deliberately polarized signals from the surrounding radio and TV towers. This arrangement would work best if the antennas were 2dimensional, so that they could be collocated at the same position at right angles.

Since there did not seem to be any conventional antenna that would meet the above specifications, it was decided to use a GA to optimize a Yagi type structure for this unconventional application. Yagi antennas are usually used for high-gain, narrowband applications. The desired bandwidth and beamwidth were very large for this kind of antenna, and the sidelobe requirements were very difficult 
to meet. However, a standard Yagi antenna is 2dimensional and therefore able to meet the polarization and collocation requirements.

The number of wires was specified to be 14 . The variables were: the length of each element (14 were required), each constrained to be symmetric and between 0 and $1.5 \lambda$, the spacing between each set of two elements (13 were required), constrained to be between $0.05 \lambda$ and $0.75 \lambda$ (the total boomlength was allowed to vary), and the diameter of the wire, constrained to be 2, 3, 4, 5 or $6 \mathrm{~mm}$. This wide latitude in parameters allowed the GA to explore very unconventional areas of the Yagi search space.

Note that of the total 28 variables, 27 of them were continuous, real-numbered parameters, making this a natural problem for a real-valued chromosome. The discrete variable-wire diameter-used a real-valued gene, but it was discretized so the GA would only use one of the allowed values. Doing so is usually not recommended for the type of crossover techniques used, but the problem was insensitive to this parameter and it did not affect results adversely.

As expected, the GA produced an antenna that approached the above requirements, though its configuration was quite unconventional for a Yagi antenna. It differed from a conventional Yagi in that the director elements were very closely spaced, its overall length was much less than a typical Yagi with the same number of elements, and its wire lengths varied haphazardly. The genetic Yagi had 13 elements (plus the

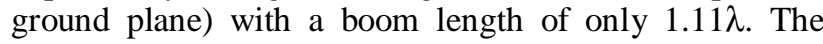
directors varied in length in a seemingly random fashion from about $0.25 \lambda$ to $0.4 \lambda$ with an average spacing of less than $0.1 \lambda$, as shown in Figure 4. A conventional 14 element Yagi has a boom length about 3 times as long, with directors that are about $0.4 \lambda$ in length and $0.35 \lambda$ in spacing, and the lengths become slightly shorter and the spacings slightly larger the greater the distance from the driven element.

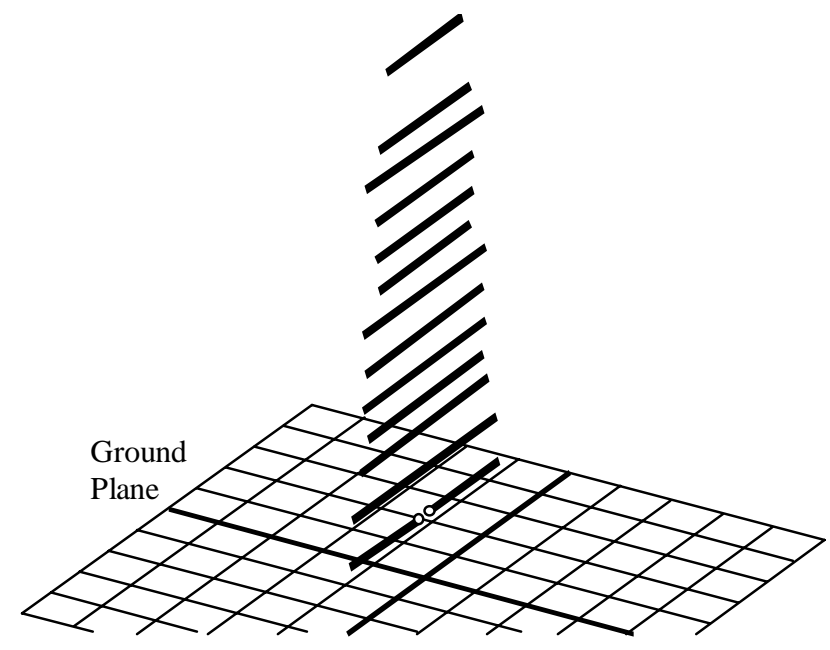

Figure 3: Genetic Yagi Feed for the Arecibo Radio Telescope. From [3].

The performance of this Yagi was computed at $2 \mathrm{MHz}$ increments over the band from 219 to $251 \mathrm{MHz}$, a bandwidth of $13.6 \%$. The figure below shows the E-plane patterns and $\mathrm{H}$-plane patterns for the genetic Yagi over a finite ground plane at the same frequencies. It is seen that the sidelobe levels for both planes are more than $25 \mathrm{~dB}$ lower than the gain at $0^{\circ}$ from 223 to $243 \mathrm{MHz}$, the most important part of the band, and are more than $20 \mathrm{~dB}$ lower over the rest of the frequency band. The E- and H-plane half-power beamwidths range from 51 to $55^{\circ}$ and 64 to $69^{\circ}$ respectively, slightly larger than desired but certainly acceptable. The VSWRs are less than 3.0 from 227 to 245 $\mathrm{MHz}$, though they are higher at the ends of the frequency band. The antenna gain ranged from 10.4 to $11.0 \mathrm{~dB}$ over the frequency band. This gain is approximately $1 \mathrm{~dB}$ lower than that for a Yagi that is optimized for maximum gain. 

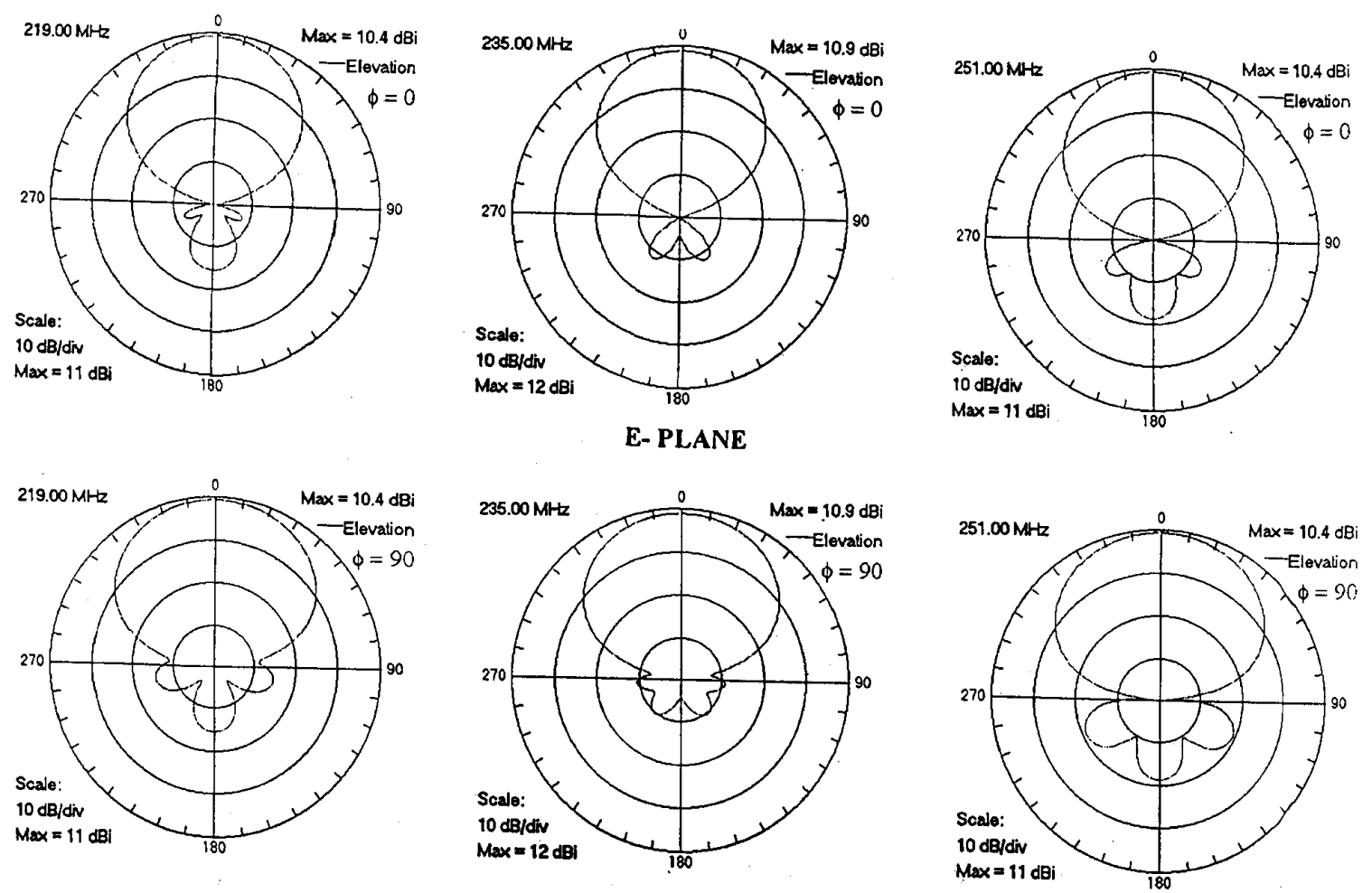

H- PLANE

Figure 4: Computed Gain Patterns of Yagi Over a Ground Plane at 219, 235 and $251 \mathrm{MHz}$. From [3].

The antenna was fabricated to a $1 / 6^{\text {th }}$ scale and the Eplane patterns and VSWR were measured. The computed and measured patterns had reasonable agreement. The measured VSWRs were less than 3.0 over most of the band and had a maximum value of 3.7 near the ends. The measured gains were slightly less than $10 \mathrm{~dB}$; however, if the reflection losses are taken into account, the corrected values for a matched antenna approach the computed gains. For more information about this antenna, see [3] and [8].

This antenna shows the power of the GA to mold conventional designs into new form to solve difficult problems. Naturally, it is important to allow the GA sufficient latitude in the design parameters to change the design from its traditional form to something new.

However, it is not always necessary to specify a design at all, in which case the GA is truly the inventor of an antenna, as the next section will show.

\section{UNCONVENTIONAL DESIGN: THE CROOKED-WIRE GENETIC ANTENNA}

The application of interest in this section is fairly conventional: ground-to-satellite communications using omnidirectional antennas. Antennas for this application, intended for use on cars or handsets, must be cheap, robust, and have as uniform a gain pattern across the hemisphere as possible for a right-hand circularlypolarized signal, excluding low elevations less than $10^{\circ}$ above the horizon, where multipath problems will arise. (Multipath refers to the reception of a signal from more than one path, such as receiving a signal from direct lineof-sight and from a reflection off the ground. When the multipath signals do not arrive in phase and at the same time, as generally happens, problems arise such as the ghosting seen on televisions.) This antenna is not trivial to design, and several conventional designs, such as the quadrafilar helix, have emerged to solve this problem to varying degrees. These antennas tend to be expensive and narrowband, and they often require a signal to be fed to the antenna in two places with a precise difference in phase to set up the circular wave. There is thus considerable room for improvement in the state of the art.

There are several qualities that one might desire such an antenna to have, which can be turned into general constraints on the design. For instance, one might desire a single feed point at the base of the antenna for simplicity and low-cost. In addition, it is helpful to have such an antenna over a ground plane. The antenna is expected to be relatively small because near-hemispherical coverage is desired, so it would make sense to constrain the search space to a fairly small volume, for instance, a cube half a wavelength on a side, with the antenna's base located in the center of the bottom face. Doing so will increase the 
average speed of simulation while having little impact on results.

It would also be convenient if the antenna were a connected series of straight wires so that there are no precision bends, floating parasitics, or branch points required, for ease in fabrication. 5, 6, 7 and 8 straight wire segments connected in series were thus chosen for investigation. (Preliminary results showed the 7-wire antenna performed slightly better than the others, so 7 wires will be used from here on.) A depiction of the search space that incorporates these constraints is shown in the figure below.

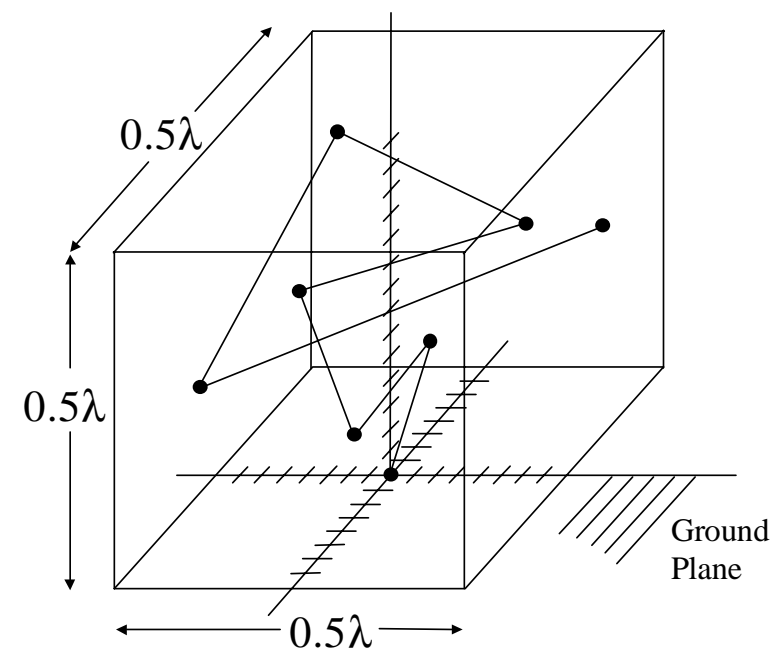

Figure 5: Crooked-Wire Genetic Antenna Search Space for 7 Wires

The GA has performed well up to this point, but it has always had a pre-existing design to use as a template for its work. Can a GA really produce a good antenna with its only engineering knowledge coming from constraints based on convenience?

To begin encoding this problem for a GA, the location of the nodes defining the start and endpoints of the wire segments were mapped into a chromosome. Since each node requires three coordinates, the 21 parameters were placed into a chromosome, i.e., Point1-X, Point1-Y, Point1-Z, Point2-X..., each value encoded into 5 binary bits. 5 bits, corresponding to 32 levels, was chosen because the accuracy of fabrication was not expected to be better than this resolution ( $3 \mathrm{~mm}$ at $1600 \mathrm{MHz})$. Thus, the whole chromosome required 105 bits.

The cost function was then determined for this antenna. The goal was to obtain right hand circular polarization $10^{\circ}$ above the horizon at a frequency of $1600 \mathrm{MHz}$. A good measure of that desired performance can be found in the sum of the squares of the deviation of all calculated gains from the mean. In equation form:
Fitness $=\Sigma_{\text {over all } \theta, \phi}(\operatorname{Gain}(\theta, \phi)-\operatorname{Avg} \text {. Gain })^{2}$.

The GA's goal was to minimize this fitness. For its first attempt at finding a 7-wire antenna, the steady-state GA had a population of 500 chromosomes, $50 \%$ overlap from generation to generation, and a $1 \%$ mutation rate. It also used one-point crossover, which was allowed to occur between any two bits in the chromosome with equal probability.

After several hours, the GA converged on a 7-wire configuration with a highly unusual shape, as shown in the inset and the photograph in the figures below. This shape was so unusual and its simulated performance so good that great care was taken to ensure its validity, including building and testing it [9].

The computed radiation patterns of the antenna over an infinite ground plane are shown below for azimuth angles of $0^{\circ}, 45^{\circ}, 90^{\circ}$ and $135^{\circ}$ at a frequency of $1600 \mathrm{MHz}$. This pattern varies by less than $4 \mathrm{~dB}$ for angles over $10^{\circ}$ above the horizon-excellent performance, especially since the antenna is so inexpensive and simple to build. (Simple is a relative term, of course, for it does not look all that simple in the figure. However, it was possible to fabricate this antenna by hand using very simple tools, while a conventional design would be tremendously difficult to manufacture by hand.)

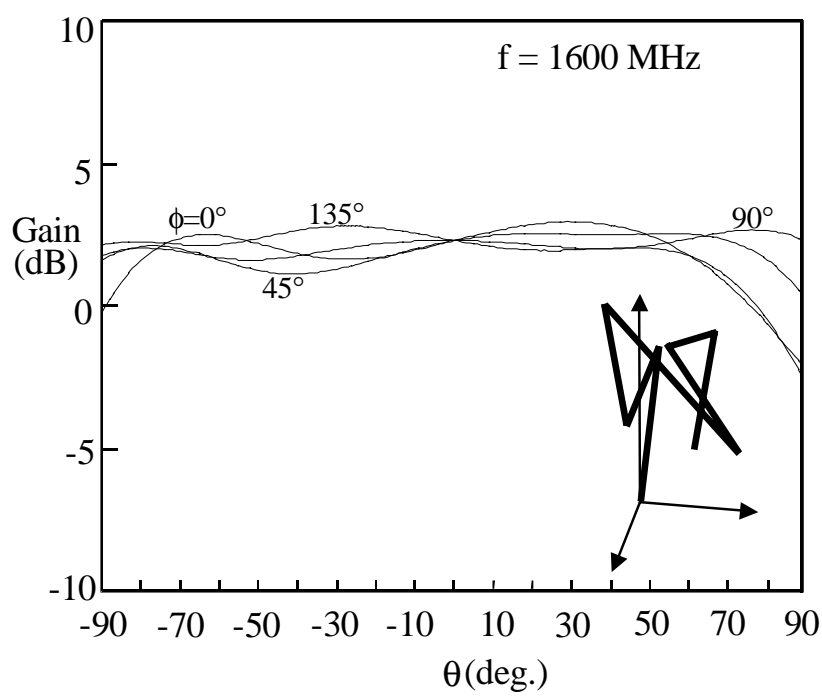

Figure 6: Crooked-Wire Genetic Antenna Radiation Pattern and Diagram. From [6]. 


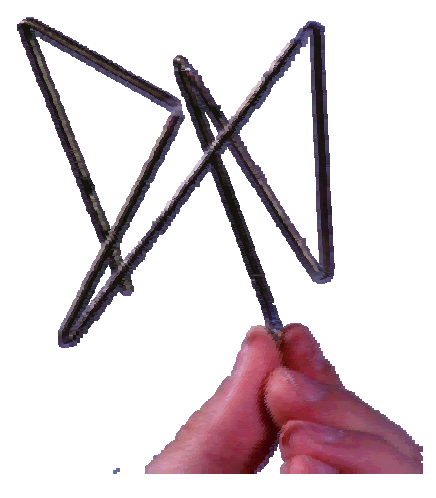

Figure 7: Photograph of the Actual Crooked-Wire Genetic Antenna. From [6].

Although this antenna was only designed to operate at a single frequency, its performance was also investigated for the range of 1300 to $1900 \mathrm{MHz}$, and it was found to have bandwidth of over $30 \%$, which is excellent for a circularly polarized antenna having near hemispherical coverage.

The antenna was built and measured for its radiation properties. There was about a $6 \mathrm{~dB}$ variation in the field above an elevation angle of $10^{\circ}$ as compared to the computed variation of about $4 \mathrm{~dB}$. This small discrepancy exists because the measurements were made over a $1.2 \mathrm{~m}$ $\mathrm{x} 1.2 \mathrm{~m}$ ground plane, while the computations were performed for an infinite ground plane. Patterns measured over the frequency range from 1300 to $1900 \mathrm{MHz}$ also compared well with the computed patterns.

After this spectacular result, many other GAs, both binary and real-valued, were run for these requirements, and the results were never the same [6]. Two more antennas optimized with the same constraints, chromosome and fitness function are shown below. Though they have nearly the same performance, they are quite dissimilar in shape from each other and from the antenna above. From these and other runs it is apparent that this search space is highly multi-modal, with many minima that give similar performance [6].

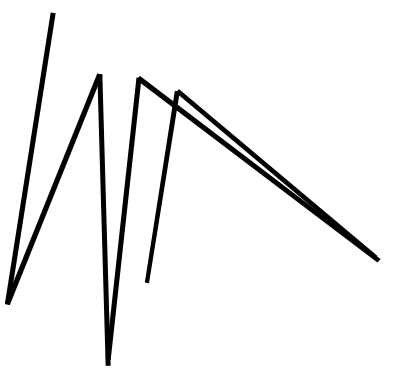

Antenna 2

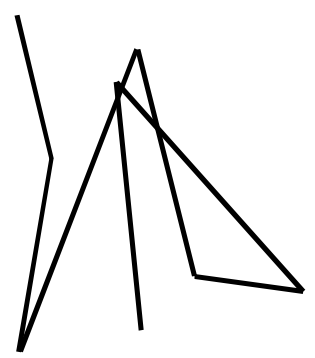

Antenna 3
Figure 8: Two More Crooked-Wire Antennas with Nearly Identical Performance

Because of the revolutionary nature of this antenna design process, a patent has been awarded [14]. This patent, which appears to be the first of its kind [15], covers the process of creating a new antenna using no previously known underlying theory of operation and the antennas created by this process.

Though patented by people, the antennas are the innovation of the GA, for it does not have much useful configuration input from the designer, and any constraints which it does have are made for convenience rather than for antenna design soundness.

The design in Figure 7 was the first genetic antenna, and though it has been in existence since 1995, it is an excellent demonstration of the power of the GA. Ongoing research has greatly advanced the state of the art of the genetic antenna, however. It has been applied with great success to several other problems, to include very small antennas [10], uniform gain for low elevation angles over a lossy ground [11], and adaptation of an antenna to its environment for omnidirectional and high-gain applications [12]. For more information about this antenna and other antennas optimized by GA, see also [1, $3,8,9,13]$.

\section{CONCLUSION}

Each of the antennas described above demonstrated a different quality of GAs as applied to wire antenna design. The Yagi antenna optimized for the Arecibo Feed problem shows how the GA can change conventional designs, using them with unusual parameters, to solve unconventional problems. The crooked-wire genetic antenna shows the raw power of the GA to find not just an optimized design for an application, but to create a new design with minimal help from the engineer.

It may seem extremely surprising that a GA can autonomously find such amazing antennas. However, consider that many people have optimized antennas without any knowledge of electromagnetic theory through the adjustment of their TV's "rabbit ears" antenna. What is used is a rather stochastic local-search technique, based on feedback from the quality of reception, sometimes 
even involving haphazard pieces of aluminum foil, to find the best reception. It is usually unknown if the television viewer has found the best possible reception, but the process is stopped once reception has been found that is "good enough" or appears unlikely to improve. The antenna configuration and characteristics are usually quite different from the original V-shaped design, but it works, at least while the surrounding conditions remain constant. In addition, many different configurations will often give the same performance.

Similarly, the GA uses feedback from the antenna simulator to search, somewhat more effectively, the large search space of antenna configurations to find one that is acceptable. As with most complex engineering problems, it is very difficult to tell if the GA has found the best antenna, but often that is not as important as having an acceptable solution. And as in the case of the television antenna, many different antenna configurations may give similar performance, depending on the problem. So while the results shown in this chapter are indeed remarkable, they are not unreasonable, given the nature of antenna design.

In summary, then, GAs are able to optimize wire antennas for diverse and difficult applications. The inherent power of the GA to not only optimize conventional designs, but to create them virtually on its own, makes it an ideal method of automated design for antennas.

\section{REFERENCES}

[1] Electromagnetic Optimization by Genetic Algorithms. Y. Rahmat-Samii and E. Michielssen, eds., Wiley, 1999.

[2] G.J. Burke and A.J. Poggio. "Numerical Electromagnetics Code (NEC)-Method of moments." Rep. UCID18834, Lawrence Livermore Laboratory, January 1981.

[3] E.E. Altshuler and D.S. Linden. "Wire Antenna Designs using a Genetic Algorithm." IEEE Antenna \& Propagation Society Magazine, Vol. 39, pp. 33-43, April 1997.

[4] I.M. Avruch, et al., "A Spectroscopic Search for Protoclusters at High Redshift." Bulletin of the American Astron. Society, Vol. 27, No. 4, 1995.

[5] A. Adewuya, "New Methods in Genetic Search with Real-valued Chromosomes," Master's Thesis, Mech. Engr. Dept., MIT, 1996.

[6] D.S. Linden. "Automated Design and Optimization of Wire Antennas using Genetic Algorithms." Ph.D. Thesis, MIT, September 1997.

[7] D.S. Linden. "Using a Real Chromosome in a Genetic Algorithm for Wire Antenna Optimization." Presented at and published in the Proceedings of the IEEE APS International Symposium, Montreal, Canada, 13-18 July 1997.
[8] E.E. Altshuler and D.S. Linden. "Design of Wire Antennas using Genetic Algorithms." Electromagnetic Optimization by Genetic Algorithms, Y. Rahmat-Samii and E. Michielssen, eds., Wiley, 1999.

[9] D.S. Linden and E.E. Altshuler. "Automating Wire Antenna Design using Genetic Algorithms.' Microwave Journal, Vol. 39, No. 3, March 1996.

[10] E.E. Altshuler. "Small Wire Antenna Design using a Genetic Algorithm." Presented at and published in the Proceedings of the URSI General Assembly, Toronto, Ontario, Canada, 13-21 August 1999.

[11] B.S. Sandlin. "A Wire Antenna Designed for Space Wave Radiation Over the Earth Using a Genetic Algorithm." Ph.D. Thesis, AFIT, 1997.

[12] D.S. Linden. "Wire Antennas Optimized in the Presence of Satellite Structures using Genetic Algorithms." To be presented at and published in the Proceedings of the IEEE Aerospace Conference, Big Sky, MT, 18-25 March 2000.

[13] D.S. Linden. "Creative Antenna Design using Evolutionary Computation." Creative Evolutionary Systems, Peter Bentley and Dave Corne, eds., 2002.

[14] E.E. Altshuler and D.S. Linden. "A Process for the Design of Antennas using Genetic Algorithms." Patent \#5,719,794, 17 February 1998.

[15] P. Weiss. “On the Origin of Circuits," Science News, Volume 156, pp. 156-158, September 4, 1999. 